\title{
A TWO-COLORING INEQUALITY FOR EUCLIDEAN TWO-ARRANGEMENTS
}

\author{
GUSTAVUS J. SIMMONS ${ }^{1}$ AND JOHN E. WETZEL
}

\begin{abstract}
We prove that for any properly two-colored arrangement of lines in the Euclidean plane having, say, $r$ red and $g$ green regions with $r>g$, the inequality

$$
r<2 g-2-\sum_{P}(\lambda(P)-2)
$$

holds, where for each point $P$ of intersection of the lines, $\lambda(P)$ is the number of lines of the arrangement that contain $P$. This strengthens recent results of Simmons and Grünbaum.
\end{abstract}

It is easy to show by induction that the regions formed by $n$ lines in the Euclidean plane can be colored with two colors in such a way that contiguous regions have different colors. Suppose there are $r$ red regions and $g$ green regions, with $r \geqslant g$. In 1972, Simmons presented a proof of the inequality

$$
r \leqslant 2 g-2
$$

for simple arrangements (in the projective plane) at a Mathematical Association of America meeting [5], but that proof has never been published. In 1973, the same inequality was mentioned without proof in Simmons [6]. Grünbaum [3] gave a proof of (1) for arbitrary nontrivial arrangements of lines in the Euclidean plane. In this note we prove a stronger inequality of a similar kind for arbitrary Euclidean arrangements.

Suppose a properly two-colored arrangement of $n \geq 2$ lines is given in the Euclidean plane. If the lines are all parallel, it is easy to see that (1) holds provided $n \neq 2$ and $n \neq 4$; and the equality in (1) holds if and only if $n=3$ or $n=6$. From now on we suppose the lines are not parallel.

Let $\mathscr{P}$ be the set of points of intersection of the lines, and for each point $P$ of $\mathscr{P}$ let $\lambda(P)$ be the number of lines on $P$. It is our claim that

$$
r \leqslant 2 g-2-\sum_{P \in \mathscr{P}}(\lambda(P)-2),
$$

and the equality holds if and only if every red region is a triangle if it is bounded and a digon (i.e., an angle) if it is unbounded.

Received by the editors December 12, 1978.

AMS (MOS) subject classifications (1970). Primary 50B30; Secondary $05 \mathrm{C} 15$.

Key words and phrases. Two-colorings, two-arrangements, arrangements of lines, arrangements of pseudolines.

'The first author was supported by the U. S. Department of Energy (DOE) under contract AT(29-1)-789 (Sandia Laboratories is a DOE facility).

(C) 1979 American Mathematical Society 0002-9939/79/0000-0476/\$02.00 
Suppose there are $r_{k}^{\prime}$ bounded red regions that have $k$ sides and $r^{\prime}$ bounded red regions in all. Since there are

$$
S^{\prime}=-n+\sum_{P \in \mathscr{P}} \lambda(P)
$$

(bounded) line segments in the arrangement (see [1, p. 444]) each of which abuts at most one bounded red region, we see that

$$
3 r^{\prime} \leqslant \sum_{k>3} k r_{k}^{\prime} \leqslant S^{\prime}=-n+\sum_{P \in \Phi} \lambda(P) .
$$

The outermost terms in (4) are equal if and only if $r_{k}^{\prime}=0$ for $k>3$, which gives equality on the left, and every bounded line segment abuts exactly one bounded red region, which gives equality on the right. This latter condition is obviously equivalent to the demand that every unbounded red region be a digon, and so the condition for equality in (4) is precisely as stated for (2).

Because there are

$$
R^{\prime}=1-n+\sum_{P \in \mathscr{P}}(\lambda(P)-1)
$$

bounded regions (see [1, p. 444]), there must be

$$
g^{\prime}=R^{\prime}-r^{\prime} \geqslant 1-\frac{2 n}{3}+\sum_{P \in \mathscr{P}}\left(\frac{2 \lambda(P)}{3}-1\right)
$$

bounded green regions. The $2 n$ unbounded regions simply alternate in color, and so

$$
\begin{aligned}
& r=r^{\prime}+n \leqslant \frac{2 n}{3}+\sum_{P \in \mathscr{P}} \frac{\lambda(P)}{3} \\
& g=g^{\prime}+n \geqslant 1+\frac{n}{3}+\sum_{P \in \mathscr{P}}\left(\frac{2 \lambda(P)}{3}-1\right) .
\end{aligned}
$$

It follows that

$$
2 g-r \geqslant 2+\sum_{P \in \Phi}(\lambda(P)-2)
$$

which is (2). Since the equality in (2) entails the equality in (4), the assertion is completely proved.

Inequality (2) is sharp in the sense that for each $n$ there is an arrangement of $n$ lines for which the equality holds-one can simply take the " $n$-star," with all $n$ lines concurrent in a point. Less special cases of equality seem more difficult to find.

Arrangements of $n$ lines in general position for which the equality holds in Simmons' inequality (1) (and hence in (2)) are known for $n=2,3,5$, 9, and 15; the arrangement of 5 lines is formed by the sidelines of a regular pentagon, the arrangement of 9 lines is pictured in Palásti [4], and the arrangement of 15 lines was found by Simmons and appears in [6]. Equality in (2) holds for the arrangement generated by extending the sides of a regular 
$n$-gon precisely for $n=3,5$, and 6 and for the arrangement generated from the $n=\left(\begin{array}{c}m \\ 2\end{array}\right)$ lines determined by the vertices of a regular $m$-gon precisely for $m=3$ and $m=5$. It is easy to verify that equality in (2) cannot hold if some parallel family has more than two lines.

Additional arrangements with equality can clearly be obtained from other such arrangements by moving the lines so as to squeeze a red triangle down to a point. Many nontrivial arrangements with equality can be obtained in this manner from the arrangements described above. No nontrivial cases of equality in (2) involving more than 15 lines are known.

The argument actually proves (2) for connected arrangements of crosscuts in a simply-connected plane domain each two of which have at most one point in common, because formulas (3) and (5) hold for such arrangements (see [1] and [7]). In particular, inequality (2) holds for any connected arrangement of pseudolines in the Euclidean plane.

Finally, it follows from (2) that $r / g \leqslant 2$ for every properly two-colored arrangement of at least two lines (or pseudolines), and the equality holds precisely for two parallel lines (or two disjoint pseudolines) (cf. Palásti [4]). Dividing (6) by (7), we see that

$$
\frac{r}{g} \leqslant 2-\frac{6+3 \sum(\lambda(P)-2)}{3+n+\sum(2 \lambda(P)-3)},
$$

with equality as for (2). In particular, for $n$ lines in general position,

$$
\frac{r}{g} \leqslant 2-\frac{12}{n^{2}+n+6}
$$

As noted above, arrangements with equality are known for $n=2,3,5,9$, and 15 . When $n \equiv 1(\bmod 3)$, inequality $(4)$ for $n$ lines in general position asserts that

$$
r^{\prime} \leqslant \frac{1}{3}\left(n^{2}+n-2\right)
$$

because $r^{\prime}$ has to be an integer; and then Simmons' inequality (1) becomes $r<2 g-4$, and inequality (8) strengthens to

$$
\frac{r}{g} \leqslant 2-\frac{24}{n^{2}+n+10} \text {. }
$$

Arrangements with equality here are known only for $n=4$ and $n=7$. The apparently difficult question of determining the maximum of $r / g$ for each $n$ was posed by Fejes Tóth [2].

\section{REFERENCES}

1. G. L. Alexanderson and John E. Wetzel, Dissections of a plane oval, Amer. Math. Monthly 84 (1977), 442-449.

2. L. Fejes Tóth, A combinatorial problem concerning oriented lines in the plane, Amer. Math. Monthly 82 (1975), 387-389. 
3. Branko Grünbaum, Two-coloring the faces of arrangements, Period. Math. Hungar. (to appear).

4. Ilona Palásti, The ratio of black and white polygons of a map generated by general straight lines, Period. Math. Hungar. 7 (1976), 91-94.

5. G. J. Simmons, A linear partitioning of the plane which extends the known solutions of four combinatorial problems, MAA Southwestern Section annual meeting, March 24-25, 1972, Albuquerque, New Mexico (title only), Amer. Math. Monthly 79 (1972), 824.

6. A A maximal 2-arrangement of sixteen lines in the projective plane, Period. Math. Hungar. 4 (1973), 21-23.

7. John E. Wetzel, Dissections of a simply-connected plane domain, Amer. Math. Monthly 85 (1978), 660-661.

Department of Applied Mathematics, Sandia laboratories, Albuquerque, New Mexico 87185

Department of Mathematics, University of Illinois at Urbana-Champaign, Urbana, ILLINOIS 61801 